\title{
La Alienación Parental como una forma de vulnerar los Derechos de los Niños, Niñas y Adolescentes
}

\author{
Parental Alienation as a way of violating the Rights of Children and Adolescents \\ Alienação dos pais como forma de violar os Direitos da Criança e do Adolescente
}

Diego Javier Valdivieso Velasco

dvaldivieso-asociados@outlook.com

diegool_007@hotmail.com

https://orcid.org/0000-0001-7497-0796

Universidad Central del Ecuador, Quito-Ecuador

\author{
Laura Baque \\ abglaurabaque@gmail.com \\ laurabaqueandrade@outlook.com \\ https://orcid.org/0000-0003-3426-6811 \\ Universidad Central del Ecuador, Quito-Ecuador
}

Artículo recibido en octubre 2020 | Arbitraje en noviembre 2020 | Aceptación en diceimbre 2020 | Publicación en enero 2021

RESUMEN

\section{ABSTRACT}

RESUMO
La familia es el principal elemento en la vida de un niño por tal motivo es importante la protección legal en caso de divorcio, en este contexto la investigación tuvo como objetivo analizar la incidencia de la alienación parental en niños y adolescentes, su metodología fue de tipo descriptiva, bajo un diseño documental de campo, con un enfoque cualitativo, el método usado fue sistemático y analítico, la técnica e instrumento que se usaron fueron la encuesta y el cuestionario. La población y muestra estuvo conformada por 2420 padres divorciados registrado en Quito, y se aplicó el instrumento a los 242 padres que residen en esa ciudad. Se obtuvo como resultado, la alienación parental puede influir negativamente en los procesos legales de procesos de divorcio, por ende es necesario que se reconozca legalmente en el ordenamiento jurídico de Ecuador y se reforme el Código de la Niñez y la Adolescencia.

Palabras clave: Alienación parental; derechos; divorcio; familia, patria potestad

The family is the main element in the life of a child, for this reason it is important the legal protection in case of divorce, in this context the research had the objective of analyzing the incidence of parental alienation in children and adolescents, its methodology was descriptive, under a field documentary design, with a qualitative approach, the method used was systematic and analytical, the technique and instrument used were the survey and the questionnaire. The population and sample consisted of 2420 divorced fathers registered in Quito, and the instrument was applied to 242 fathers residing in that city. As a result, parental alienation can have a negative influence on the legal processes of divorce proceedings, therefore it is necessary that it be legally recognized in the legal system of Ecuador and that the Code of Childhood and Adolescence be reformed.

Key words: Parental alienation; rights; divorce; family, parental authority

A família é o principal elemento na vida de uma criança, por este motivo é importante ter proteção jurídica em caso de divórcio. Neste contexto, a pesquisa teve como objetivo analisar a incidência da alienação parental em crianças e adolescentes, sua metodologia foi descritiva, sob um desenho documental de campo, com uma abordagem qualitativa, o método utilizado foi sistemático e analítico, a técnica e o instrumento utilizado foi a pesquisa e o questionário. A população e a amostra consistia de 2420 pais divorciados registrados em Quito, e o instrumento foi aplicado a 242 pais residentes naquela cidade. Como resultado, a alienação dos pais pode ter uma influência negativa nos processos judiciais de divórcio, portanto é necessário que ela seja legalmente reconhecida no sistema jurídico do Equador e que o Código da Infância e da Adolescência seja reformado.

Palavras-chave: Alienação dos pais; direitos; divórcio; família; autoridade dos pais 


\section{INTRODUCCIÓN}

Los niños, niñas y adolescentes están protegidos legalmente por una serie de principios y derechos reconocidos tanto en instrumentos de carácter internacional como la Convención de los Derechos del niño y en el ordenamiento jurídico, mediante los preceptos constitucionales y las restantes normas de la materia, entre estos derechos se pueden mencionar: el derecho a tener una familia y disfrutar de la convivencia familiar, el derecho a ser escuchado, el derecho a disfrutar de su integridad física y psíquica, entre otros.

Estos derechos y principios deben ser aplicables en la vida cotidiana para que realmente adquieran el valor que se pretende con ellos, lo que no siempre ocurre, motivos por los cuales estos pueden ser vulnerados por determinadas personas e instituciones y entre ellos están en muchos casos sus propios progenitores, esta problemática puede manifestarse en cualquier sitio del planeta.

Cabe destacar, que la violación a los derechos de los niños, niñas y adolescentes pueden manifestar ante diferentes supuestos como: divorcio, separación, cuando los niños conviven en el mismo domicilio con ambos progenitores y en los casos de las madres solteras, al aparecer el fenómeno conocido como Alienación Parental.

Este fenómeno se describe por Gardner (1985), como: “una respuesta de contexto familiar típica al divorcio o separación de sus padres, en la cual el niño resulta alienado respecto de uno de sus progenitores y acosado con la denigración exagerada y/o injustificada del otro progenitor" (p. 62).

Por tal motivo, es importante que los derechos de los niños, niñas y adolescentes sean reconocidos constitucionalmente los cuales, ante la presencia del fenómeno y la carencia de un reconocimiento legal en el país, conlleva a que se vulneren principios elementales en materia de derechos de la niñez y la adolescencia; así como sus derechos.

En lo que respecta a la Alienación Parental en el Ecuador este fenómeno no está reconocido legalmente en el país, lo que genera que muchos niños queden en desamparo ante la presencia de este fenómeno que suele presentarse ante procesos derivados de asuntos de familia.

Por lo tanto, la inexistencia en la legislación ecuatoriana del fenómeno de la Alienación Parental, genera que el mismo se expanda y se convierta en un acto común, sin dársele el reconocimiento y el tratamiento que en el orden legal y los daños que causa a los niños en su vida presente y futura amerita, vulnerando los derechos elementales de los niños, niñas y adolescentes, lo que influye negativamente en su desarrollo integral.

En este sentido, la presente investigación, tiene como objetivo principal, analizar la incidencia de la Alienación Parental en niños, niñas y adolescentes en el Distrito Metropolitano de Quito. Siendo ésta de gran importancia desde el punto de vista social y legal, puesto que la Alienación 
Parental afecta a miles de niños, niñas y adolescentes tanto a nivel internacional como nacional, ya que, constituye un fenómeno que de no reconocerse y manejarse de la forma correcta trae consigo consecuencias en el orden jurídico y familiar irreparables causando un gran impacto negativo en la sociedad ecuatoriana.

\section{Alienación parental}

Los derechos de los niños, niñas y adolescentes han pasado por una etapa de evolución en cuanto sus derechos, puesto que inicialmente no eran considerados sujetos de ellos lo que traía consigo para ellos serias dificultades y una desprotección total, la Convención sobre los Derechos del Niño (1990) jugó un papel elemental para darles el lugar que legalmente merecen con el fin de asegurar la tutela de estos, quedando establecido en dicho instrumento desde su preámbulo que el niño aún carece de madurez física y mental por lo que necesitan toda la protección y cuidado así como la debida protección legal desde el momento que se concibe y después de haber nacido, por lo tanto se debe, "asegurar al niño la protección y el cuidado que sean necesarios para su bienestar, teniendo en cuenta los derechos y deberes de sus padres, tutores u otras personas responsables de él"(Convención sobre los Derechos del Niño, 1990, p. 2).

Ante los diferentes eventos de la vida familiar en los que los niños, niñas ya adolescentes se ven involucrados como: el divorcio, separación de sus padres y conflictos internos que se pueden manifestar tanto fuera como dentro del marco de su domicilio se comenzó a manifestar un fenómeno, Gardner (1985), lo denomina como Alineación Parental, el cual es:

Un trastorno que surge principalmente en el contexto de las disputas por la guarda y custodia de los niños. Su primera manifestación es una campaña de difamación contra uno de los padres por parte del hijo, campaña que no tiene justificación. El fenómeno resulta de la combinación del sistemático adoctrinamiento (lavado de cerebro) de uno de los padres y de las propias contribuciones del niño dirigidas a la denigración del progenitor objetivo de la de la campaña. (p. 6).

Aguilar (2013) considera el fenómeno como un trastorno que presenta varios síntomas y da lugar a que el progenitor influya en la conciencia de sus hijos utilizando artimañas, estrategias dirigidas a obstaculizar la relación e incluso a destruir los vínculos con el otro progenitor.

Francois (2001), lo define como un proceso que realiza el progenitor (a) consistente en programar una conducta de rechazo al otro progenitor (b) sin una justificación objetiva (p. 4).

Así que, se puede afirmar que son los niños, niñas y adolescentes el objeto de la Alienación Parental pues es el vehículo que usa uno de los padres para afectar en muchas ocasiones 
de manera irreversible la imagen del otro. En el fenómeno intervienen dos elementos, el denominado progenitor alienante que es el que activamente incide en que el niño rechace a su otro padre y el progenitor alienado quien es una víctima pues se lesiona y denigra su imagen hasta llegar a que sus hijos lo rechacen. Esta situación da lugar a la aparición en los niños, niñas y adolescentes de daños emocionales y psicológicos que pueden afectarlos transitoriamente o de forma definitiva, impidiendo en todos los ámbitos el normal desarrollo de estos.

Por otro lado, Zariñan (2015), analiza este fenómeno muy vinculado a la vulneración de los derechos elementales de los niños, niñas y adolescentes y trae como consecuencia un abuso de poder por parte de uno de los padres para lograr sus fines de dañar el honor, imagen y credibilidad del otro progenitor.

Este fenómeno como se ha argumentado es motivado por conflictos de legales, se puede poner de manifiestos en los diferentes ámbitos de la vida tal y como se ha explicado anteriormente, se destaca porque uno de los padres pone al niño en contra del otro progenitor y vulnera los derechos básicos de los niños, niñas y adolescentes tanto los reconocidos en instrumentos internacionales como en los instrumentos de orden internacional.

\section{Principio de Interés Superior del Niño}

La Corte Interamericana de Derechos Humanos (2009), reconoce el principio basado en la dignidad del ser humano, en las características de los niños, niñas y adolescentes y en la necesidad de asegurar el desarrollo de éstos, aprovechando todas sus potencialidades. En virtud de lo antes expuesto este principio protege los derechos fundamentales de las niñas, niños y adolescentes.

Por otro lado, el Comité de Naciones Unidas de los Derechos del Niño (2013) ha establecido varios aspectos los que deben interrelacionarse armónicamente para materializar el principio de interés superior del niño ellos son: su opinión, identidad, la conservación del entorno familiar y el mantenimiento de las relaciones entre sus miembros, el cuidado, protección y seguridad que debe brindársele a los niños, la situación de vulnerabilidad y los derechos relacionado con la salud y la educación.

\section{Derecho a vivir en familia}

La familia es el principal elemento en la vida de un niño, con el fin de conservar estos lazos familiares al existir un divorcio, separación u otras formas de relaciones entre padres e hijos, se establecen en los Juzgados de familia los procesos legales encaminados a determinar la tenencia de los niños, el régimen de visitas, la patria potestad y los procesos de alimentos, pero al aparecer una tendencia malsana por parte de uno de los progenitores contra el otro, ya estas disposiciones carecen del valor y de la intención legal y social con que se establecen. 
Carbonell (2008), expresó sobre la familia que:

El disfrute mutuo de la convivencia entre padres e hijos constituye un elemento fundamental en la vida de familia y una manifestación del derecho del niño a tener una familia y no ser separado de ella, aun cuando los padres estén separados (p.82).

La familia debe ofrecer al niño alimentación, descanso, higiene, cuidados, afecto, apoyo, seguridad, debe brindarle todo tipo de atención y resolver las necesidades que esta sufra, además de ser un factor esencial en la formación de su personalidad, valores y en el aprendizaje. Ambos padres deben ser corresponsables de los cuidados del niño, pero cuando el padre custodio interfiere y limita la relación de sus hijos con el otro progenitor y por ende se manifiesta la Alienación Parental, impide el cumplimiento de los deberes de los padres impidiendo darle la atención y cuidado que se le debe brindar a los niños, niñas y adolescentes, para su formación y desarrollo.

Es importante que el Estado garantice y prevenga mediante las normas legales correspondientes que ningún miembro de la familia utilice o manipule a los niños, niñas y adolescentes en contra de uno de sus progenitores, pues ello va en detrimento de su formación, desarrollo, salud mental y de todos los derechos y principios que poseen.

\section{El derecho del niño a ser consultado}

El derecho del niño a ser consultado es un derecho elemental que deben ejercer los niños, niñas y adolescentes, el mismo esta interrelacionado con el principio de interés superior del niño y con la Alienación Parental, ya que el niño en el momento de emitir un criterio u opinión relacionado con algún conflicto de familia en el que esté involucrado puede sacar a la luz sus sentimientos, intereses, si está presionado por sus progenitores o familiares cercanos y por ende permite valorar si este derecho está siendo vulnerado.

Este importante derecho está consagrado al igual que los restantes derechos en normativas internacionales y nacionales. Para estudiarlo debe tomarse como base el artículo 12 de la Convención de los Derechos del Niño (1989), la que establece:

Los Estados Partes garantizarán al niño que esté en condiciones de formarse un juicio propio el derecho de expresar su opinión libremente en todos los asuntos que afectan al niño, teniéndose debidamente en cuenta las opiniones del niño, en función de la edad y madurez del niño. Con tal fin, se dará en particular al niño oportunidad de ser escuchado, en todo procedimiento judicial o administrativo que afecte al niño, ya sea directamente o por medio de un representante o de un órgano apropiado, en consonancia con las normas de procedimiento de la ley nacional. (p. 4). 
Sobre este derecho, Simón (2008), analiza que el ejercicio de este derecho contribuye al desarrollo psíquico de los niños, niñas y adolescentes, y los forma para enfrentar la vida y vivir en sociedad, asegura que este derecho representa el respeto a sus derechos humanos.

Albán (2010) comentó que este derecho está presente en los procesos legales o administrativos que afecte a los niños y adolescentes, cuya opinión se tendrá en cuenta según la edad del niño. En el Ecuador es obligatorio escuchar a los niños mayores de 12 al momento de definir la tenencia y las reglas de comunicación y los niños y niñas menores será facultativo del juez escucharlos en los procesos y la decisión estará en función de la edad que tengan.

La Alienación Parental se puede detectar al ejercerse este derecho por los niños, ante cualquier indicio que muestre que el niño, niña o adolescente está inseguro, cohibido, debe procederse a una evaluación psicológica por expertos para determinarlo, ya que el progenitor alienante hace creer a sus hijos eventos que nunca han ocurrido y el niño al ser escuchado puede expresarlas y darlas por reales, además siempre le dará la razón y preferirá al progenitor alienante que en la generalidad es con el que convive.

\section{Patria potestad}

El Código de la Niñez y Adolescencia (2014), en el artículo 105, se reconoce la patria potestad no solamente como el grupo de derechos, sino también como las obligaciones que debe tener los padres relativos a sus hijos e hijas no emancipados, referentes al cuidado, educación, desarrollo integral, y las restantes garantías de los hijos establecidas en el ordenamiento jurídico.

Además, el artículo 106 del Código de la Niñez y Adolescencia (2014), define las reglas para confiar el ejercicio de la patria potestad las cuales se basan en los casos previstos en el artículo 325 del Código Civil y el juez luego de haber escuchado al niño, niña o adolescente que cuente con las condiciones de expresar su criterio tendrá en cuenta entre otras.

\section{Régimen de Visitas}

Este derecho de visitas en virtud de lo regulado en el artículo 123 del Código de la Niñez y la Adolescencia (2014), se hace extensivo, pues lo pueden disfrutar los parientes cercanos al niño, abuelos, tíos, primos, hermanos e incluso otras personas que aunque no guarden parentesco con los menores, están ligadas afectivamente a él, siempre que se garantice seguridad y se facilite logrando una debida armonía familiar.

Por otro lado, el artículo 122 del Código de la niñez y la adolescencia (2014), establece lo siguiente con respecto al régimen de visitas: 
En todos los casos en que el Juez confíe la tenencia o el ejercicio de la patria potestad a uno de los progenitores, deberá regular el régimen de las visitas que el otro podrá hacer al hijo o hija. Cuando se hubiere decretado alguna medida de protección a favor del hijo o la hija por causa de violencia física, sicológica o sexual, el Juez podrá negar el régimen de visitas respecto del progenitor agresor, o regular las visitas en forma dirigida, según la gravedad de la violencia. El Juez considerará esta limitación cuando exista violencia intra -familiar. Las medidas tomadas buscarán superar las causas que determinaron la suspensión. (p. 23).

Como se manifiesta en el artículo citado, el derecho a visita también podrá ser negado si concurre una de las causales descrita, siendo esta una de las vías para proteger a los niños, niñas y adolescentes, sin embargo, dentro de la violencia sicológica podría incluirse el fenómeno de estudio de ser reconocido.

MÉTODO

La metodología usada para el desarrollo del estudio, fue de tipo de descriptivo, dado a que se describió el fenómeno de la Alienación Parental, para establecer sus principales características y grados, así como para describir los derechos de los niños, niñas y adolescentes que se estudian y el principio de interés superior del niño.

Adicional el diseño de la investigación fue de tipo documental de campo. La primera porque se recopiló información existente tanto en el orden internacional como nacional sobre el tema, basado en estudios doctrinales realizados por psiquiatras, psicólogos, juristas, entre otras que obran en libros, artículos, documentos, ensayos y estudios académicos que permitieron conocer y evaluar las diferentes definiciones, teorías, grados y características sobre la investigación. Además, se realizó el análisis de la legislación nacional que permitió determinar las bases legales existentes e inexistentes sobre el fenómeno de la Alienación Parental. Y la segunda, porque este método permitió evaluar objetivamente y desde una perspectiva real el fenómeno de la Alienación Parental y su necesidad de reconocimiento.

El enfoque de la investigación fue de tipo cualitativo, puesto que se hizo un análisis de la Alienación Parental con el fin de lograr una definición completa que permitiera identificar este fenómeno, conocer y comprender la necesidad de que se reconociera legalmente en el Ecuador, además se buscó una solución jurídica con el fin de prevenirlo y eliminarlo.

Los métodos usados en la investigación fueron sistemáticos y el analítico, el primero, porque se estudió, para arribar a la necesidad e importancia de reconocer la Alienación Parental y evitar cualquier tipo de vulneración de los derechos de los niños, niñas y adolescentes. Y el segundo, porque permitió analizar la situación real y el impacto que tiene el tema en los niños, niñas, adolescentes, padres alienados y la sociedad en general.

Además, la técnica que se utilizó en el estudio fue la encuesta la cual constituyó una vía rápida y certera de recopilar información, se le aplicó a un grupo de 242 padres divorciados que han sido víctimas de manifestaciones de la Alienación Parental.

El formulario se aplicó a 242 padres que residen en el Distrito Metropolitano de Quito y se encuentran separados de sus hijos y han sido alienados, lo que se pretende con ella es demostrar 
la existencia y manifestación del fenómeno de la Alienación Parental y sus consecuencias. Siendo de esta forma, la muestra utilizada para la recolección de datos, de una población total de 4790 divorcios de los cuales corresponde un $50.52 \%$ a divorcios con hijos lo que da un total de 2420 padres divorciados (según los resultado de los registros realizados sobre estos hechos vitales en las oficinas de Registro Civil Identificación y Cedulación de la Ciudad de Quito).

\section{RESULTADO}

A continuación, serán analizados los resultados a que se ha arribado en virtud de las encuestas aplicadas a 242 padres divorciados que fueron víctimas de manifestaciones de Alienación Parental y que residen en el Distrito Metropolitano de Quito.

\section{Ítems 1. Proceso judicial derivado del divorcio o separación}

Se puede apreciar en la Tabla 1 , que $88 \%$ de los padres encuestados, han sido partes de procesos judiciales derivados del divorcio o separación que son algunos de los supuestos en que nace el fenómeno de la Alienación Parental. De ellos 29 que corresponde al 12 \% no han sido parte de estos procesos legales.

Tabla 1. Proceso judicial (Alimentos, régimen de visitas, otros).

\begin{tabular}{ccc}
\hline ALTERNATIVAS & FRECUENCIA & $\%$ \\
\hline $\mathrm{Si}$ & 213 & 88 \\
No & 29 & 12 \\
TOTAL & $\mathbf{2 4 2}$ & $\mathbf{1 0 0}$ \\
\hline
\end{tabular}

En este sentido, la mayoría de los padres divorciados, fueron víctimas de manifestaciones de Alienación Parental, por lo que se considera importante incluir en la legislación ecuatoriana estamentos que minimicen este fenómeno en padres separados o divorciados, y así evitar daños psicológicos y emocionales en los niños, niñas y adolescentes.

\section{Ítems 2. Relación padre e hijo antes del divorcio}

En la Tabla 2 se muestra que las relaciones de los padres separados con sus hijos antes de ocurrir el divorcio son en su mayoría buenas para 176 de los encuestados, representando al 73 $\%, 51$ de ellos consideran que la relación era regular lo que corresponde al 21 \% y solo 15 de ellos que representan el $6 \%$ plantean haber tenido una a mala relación con sus hijos.

Tabla 2. Relación padre e hijo.

\begin{tabular}{ccc}
\hline ALTERNATIVAS & FRECUENCIA & $\%$ \\
\hline Buena & 176 & $\mathbf{7 3} \%$ \\
Regular & 51 & $21 \%$ \\
Mala & 15 & $6 \%$ \\
TOTAL & $\mathbf{2 4 2}$ & $\mathbf{1 0 0}$ \\
\hline
\end{tabular}


Se pudo demostrar que la mayoría de los padres encuestados tenían una buena relación con sus hijos antes de divorciarse o separarse.

\section{Ítems 3. Relación padre e hijo después del divorcio}

En la Tabla 3 se muestra cómo era la relación de padres e hijos después del divorcio y separación, de ellos 39 que corresponde a un 16\% señalaron que tiene una buena relación con sus hijos, 137 de los padres encuestados expresaron tener una relación regular con sus hijos luego de la separación o divorcio, ello representa el 57 \% y 66 padres consideraron la relación que mantienen con sus hijos como mala, representando el $27 \%$.

Tabla 3. Relación padre e hijo (post).

\begin{tabular}{ccc}
\hline ALTERNATIVAS & FRECUENCIA & $\%$ \\
\hline Buena & 39 & $16 \%$ \\
Regular & 137 & $57 \%$ \\
Mala & 66 & $27 \%$ \\
TOTAL & $\mathbf{2 4 2}$ & $\mathbf{1 0 0}$ \\
\hline
\end{tabular}

Se deduce que, la mayoría de los padres no tienen una relación buena ni satisfactoria con sus hijos luego del divorcio creando así, una distancia y dificultades entre ellos motivadas por la Alienación Parental.

\section{Ítems 4. Relaciones con el padre que tiene la tenencia de su hijo}

En cuanto a este ítems, en la Tabla 4 se muestra, que ninguno de los encuestados mantiene una buena relación con el padre que posee la custodia de los niños, 185 de ellos, es decir un 76\% considera que la relación es regular y 57 manifiestan abiertamente que la relación es mala, lo que corresponde a un $24 \%$.

Tabla 4. Relación padre e hijo (post).

\begin{tabular}{ccc}
\hline ALTERNATIVAS & FRECUENCIA & $\%$ \\
\hline Buena & 0 & 0 \\
Regular & 185 & 76 \\
Mala & 57 & 24 \\
TOTAL & $\mathbf{2 4 2}$ & $\mathbf{1 0 0}$ \\
\hline
\end{tabular}

En este contexto, se evidenció que al ser malas o regulares las relaciones entre los padres, resulta imposible que ellos puedan contribuir satisfactoriamente con el desarrollo integral de los niños, niñas y adolescentes, además no se puede cumplir el derecho elemental de los niños a vivir en familia ya que estas malas relaciones frenan el ejercicio de este derecho y se puede estar en presencia de la Alienación Parental. 


\section{Ítems 5. Daño psicológico en el niño después del divorcio de los padres}

En la Tabla 5 se aprecia que el 192 de los encuestados contestó que sus hijos si han sufrido daños psicológicos luego del divorcio y separación, estas cifras representan un $79 \%$ de los encuestados y solo 50 padres correspondiendo al 21 \% consideran que sus hijos no han sufrido de esta afectación.

Tabla 5. Daño psicológico en los hijos de padres divorciados.

\begin{tabular}{ccc}
\hline ALTERNATIVAS & FRECUENCIA & $\%$ \\
\hline Si & 192 & 79 \\
No & 50 & 21 \\
TOTAL & $\mathbf{2 4 2}$ & $\mathbf{1 0 0}$ \\
\hline
\end{tabular}

De esta manera, se pudo sintetizar que la mayoría de los padres mantenían una relación buena con sus hijos antes del divorcio o separación, situación que cambia al ocurrir estos eventos. Además, el $100 \%$ de los encuestados consideraron que el padre que posee la tenencia de los niños, niñas o adolescentes influye en las malas o regulares relaciones que comienzan a manifestar hacia ellos. A su vez, muchos de los niños de los entrevistados han sufrido daños psicológicos a raíz del divorcio o separación.

La encuesta aplicada demostró la necesidad de reconocer el fenómeno de la Alienación Parental en la legislación ecuatoriana, puesto que este fenómeno es transgresor del principio de interés general del niño, y vulneran los derechos elementales de estos, por lo que deben aplicarse sanciones civiles y administrativas a los padres que incurran en la Alienación Parental, basado en todo lo antes expuesto resulta urgente y necesario realizar modificaciones al Código de la Niñez y la Adolescencia, en cuanto a incluir el fenómeno de la Alienación Parental dentro de las causas de suspensión y privación de la patria potestad.

\section{Propuesta de proyecto de reforma a los artículos 112 y 113 del Código de la Niñez y la Adolescencia referente a la suspensión y a la privación o perdida de la patria potestad}

La modificación del Código de la Niñez y Adolescencia, en cuanto a reconocer el fenómeno de la Alienación Parental y a que su presencia sea causa de suspensión, perdida o privación judicial de la patria potestad permitirá cumplir con el principio de interés superior del niño y el respeto a los derechos de los niños, niñas y adolescentes.

\section{Objetivo de la propuesta}

- Evitar la vulneración de los derechos elementales de los niños, niñas y adolescentes reconocidos en instrumentos internacionales y en la Constitución de la República del Ecuador. 
- Cumplir con el principio de interés superior del niño.

- Imponer sanciones civiles a aquellos padres que incurran en la Alienación Parental.

\section{Descripción de la propuesta}

\section{Exposicion de motivos}

\section{EL PLENo de LA A SAMBLEA NACIONALCONSIDERANDO:}

Que, el Ecuador es un Estado Constitucional de derechos y justicia social, democrática, soberana, independiente. unitaria, intercultural, plurinacional y laico, según lo determinael articulo 1 de la Constitución de la República del Ecuador;

Que, el numeral 9 del articula 11 de la Constisución de la República del Ecuador de fecha 20 de octubre de 2008 reconoce que: "El mass alto deber del Estado consiste en respetar y hacer respetar los derechos garantizados en la Constitución.

Que, el articulo 44 de la Constitución establece que el Estado, la sociedad y la familia promoverán de forma pricritaria el desartolo integral de las niñas, nin̄os y adolescentes, y asegurarain el ejetcicio pleno de sus derechos; se atenderá al principio de interés superior y sus derechos prevalecerán sobre bes de las demás personas.

Que, el articulo 45 del texto constitucional regula que las niflas, niffos y adolescentes gozarản de los derechos comunes del ser humano, además de los especificos de su edad. El Estado reconocera y garartizata la vida, incluido el cuidado y protección desde la concepción. Las nif̂as, nifíos y adolescentes tienen derecho a la integridad fisica y psiquica; a su identidad, nombre y ciudadania; a la salud integral y nutricién; a la educación y cultura, al deporte y recreación; a la seguridad social; a tener una familia ydisfrutar de la convivencia familiar y comunitaria; a la participación sccial; al respeto de su libertad y dignidad; a ser consultados en los asuntos que les afecten; a educarse de manera prioritaria en su idioma y en los contextos culturales propios de sus pueblos y nacionalidades; y a recibir información acerca de sus progenitores o familiares ausentes, salvo que fuera perjudicial para su bienestar.

Que, el articulo 1 del CŚdigo Orgánico de la Nâfez y Adolescencia, establece: Este Código dispone scbre la protección integral que el Estado, la sociedad y la famila deben garantizar a todos los nifios, nif́as y adolescentes que viven en el Ecuador, con el fin de lograr su desarrollo integral y el disfrute pleno de sus derechos, en un marco de libertad, digridad y equidad.

Que luego de analcrado en la Comisión de la Nrinez y Mdolescencia, el fenómeno de la Nienación Parental trae serias consecuencias psicológicas para los niffos, niffas yadolescentes y por tanto vulnera sus derechos, se ha determinado que sea reconocido enel Código de la Nínez y Adolescencia como medida de preventiva y para lograr la eliminación que este sea considerado como causa de suspensión y privación de la patriapotestad.

En uso de sus atrbuciones constitucionales y legales expide lo siguiente:

Refórmese el contenido del articulo 112 del Código Otgánioo de la Niflez y Adolescencia en cuanto a la suspensión de la patria potestad, agregando al mismo la siguiente causa:

8.- Presencia del fenómeno de la Nienación Parental en su grado leve o moderado.

Refórmese el contenido del articulo 113 del Códipo Orgánico de la Nifiez y Adolescencia en cuanto a la Privación o pérdida judicial de la patria potestad, agregando al mismo la siguiente causa:

8.- Presencia del fenómeno de la Alenación Parental en su grado más sevvero (grave). 


\section{Análisis de factibilidad de implementación de la propuesta}

El análisis de factibilidad constituye un instrumento en la toma de decisión para determinar si se debe seguir adelante con la reforma o no. La comprensión objetiva y cuantitativa de la factibilidad del proyecto propuesto es un elemento esencial para su continuidad y aceptación. Las herramientas de análisis y evaluación del proyecto son útiles para los propósitos mencionados anteriormente.

\section{Factibilidad}

\section{Factibilidad interna}

Este tipo de factibilidad se basa en la posibilidad cierta de realizar la modificación de una norma legal del sistema jurídico ecuatoriano, para cuyos efectos es vital tener en cuenta los siguientes aspectos:

- La existencia de un Estado constitucional desde el año 2008, en virtud de la nueva Constitución del Ecuador; $y$,

- Que, las normas jurídicas deben estar en consonancia y ser vivo reflejo de la sociedad actual y de los instrumentos internacionales de los cuales el país es parte, así como de los preceptos constitucionales establecidos, ya que no debe existir ningún tipo de incompatibilidad jurídica entre la norma constitucional y la infra constitucional, determina que la factibilidad interna se encuentra plenamente justificada, razón por la cual hace procedente la reforma a los artículos 112 y 113 del Código de la Niñez y Adolescencia.

\section{Factibilidad externa}

En cuanto a este tipo de factibilidad es importante señalar que los procesos de reforma de un Código, están sujetos a un procedimiento establecido en la Constitución de la República, que comienza en la formulación del proyecto, la autorización del Consejo de Administración Legislativa, realización de un primer debate, un segundo Debate, veto presidencial, allanamiento al veto y publicación en el Registro Oficial. Consideramos que, desde éste aspecto de factibilidad, es posible llevar adelante el proyecto de reforma.

\section{Impactos en el área social}

Esta área tiene relación con una sociedad y en especial los niños, niñas y adolescentes que se verán beneficiados con la aplicación correcta y adecuada de una norma jurídica que con la propuesta efectuada garantiza el cumplimiento del principio de interés superior del niño y el respeto a los derechos de los niños, niñas y adolescentes. 


\section{Impactos en el área académica}

Con esta reforma se pretende que se modifique en el Código de la Niñez y Adolescencia en sus artículos 112 y 113 incluyendo el fenómeno de la Alienación Parental como causal de suspensión, perdida o privación judicial de la patria potestad constituyendo esta reforma por su importancia un aporte de la Universidad al derecho, la sociedad y a la vida plena y desarrollo integral de los niños, niñas y adolescentes ecuatorianos

CONCLUSIONES

Al culminar el estudio referente al fenómeno de la Alienación Parental como una forma de vulnerar los derechos de los niños, niñas y adolescentes en el Distrito Metropolitano de Quito y en base a los resultados obtenidos se concluye:

El fenómeno de la Alienación Parental está definido de manera general como aquella manipulación, influencia de tipo negativa que ejerce uno de los progenitores sobre el niño con el fin denigrar la imagen del otro padre, para que el niño lo rechace, obstaculizando las relaciones entre ambos. Este fenómeno se manifiesta de forma común dentro de la sociedad ecuatoriana.

La Alienación Parental se pone de manifiesto en los supuestos en que uno de los progenitores se encuentre separado de su hijo ya sea motivado por divorcio, separación u otras causas. Este fenómeno causa serias afectaciones en el orden emocional y psíquico a los niños, niñas y adolescentes afectando su desarrollo integral.

Ha quedado demostrado en la investigación y mediante las encuestas realizadas que la Alienación Parental existe en el Ecuador e incide en la vulneración de los derechos básicos de los niños, niñas y adolescentes en el Distrito Metropolitano de Quito, especialmente en el derecho a vivir en familia, a su desarrollo psíquico e integral y el principio de interés superior del niño.

La Alienación Parental puede influir negativamente en los procesos legales de procesos de divorcio, separación y definición de las relaciones entre padres e hijos, especialmente en la tenencia, régimen de visitas y patria potestad, puesto que el niño al estar manipulado por uno de sus padres su opinión siempre la dará a favor de este, por lo que ello puede influir negativamente en la decisión del proceso y vulnerarse el principio de interés superior del niño y sus derechos, al emitirse la resolución correspondiente.

Que se reconozca legalmente la Alienación Parental en el ordenamiento jurídico del país y se reforme el Código de la Niñez y la Adolescencia en el que debe quedar previsto la aplicación de la sanción de carácter civil consistente en la suspensión de la patria potestad o la perdida de la misma a los padres que incurran en este acto. 


\section{REFERENCIAS}

Aguilar, J. (2013). Síndrome de alienación parental: Hijos manipulados o un cónyuge para odiar al otro. Madrid. Almuzara

Albán, F. (2010). Derechos de la Niñez y la Adolescencia. Tercera Edición Actualizada, Corregida y Aumentada. Quito. Cevallos

Carbonell, M. (2008). Familia, Constitución y derechos fundamentales. México D.F

Instituto de Investigaciones Jurídicas de la Universidad Autónoma de México

Código Civil del Ecuador. (2005).Registro Oficial 46

Constitución de la República. (2008). Registro Oficial No. 449

Convención de los derechos del Niño. (1989). Asamblea General de la ONU

Francois, P. (2001). El síndrome de la Alienación Parental. Psicología Jurídica. Madrid. Trotta
Gardner, R. (1985). El síndrome de Alienación Parental. Segunda edición. New York. Addendum

Régimen de visitas, 17203-2015-03960 (Unidad Judicial Especializada Tercera de la Familia, Mujer, Niñez y Adolescencia del Cantón Quito de la Provincia de Pichincha 22 de abril de 2015)

Simón, F. (2008). Derechos de la niñez y adolescencia: De la Convención sobre los Derechos del niño a las Legislaciones Integrales, Tomo 1. Quito. Editorial jurídica Cevallos

Zariñan, M. (2015). La Alienación Parental. México D.F. Comisión Nacional de Derechos Humanos 